\title{
The Application of PID Controller in Missile Longitudinal Loop System and Its Simulation
}

\author{
Bo Zhang ${ }^{\mathrm{a}^{*}}$ Qixing Lv and Yuqing Lei \\ College of Engineering, Bohai University, Liaoning, 121013, China. \\ ajzhzhb@sina.com
}

\begin{abstract}
KeyWords: Missile longitudinal loop; PID controller; Stable; Stimulation; Aerodynamics
Abstract. In the traditional missile longitudinal loop design, the additional pitch angle feedback, trajectory inclination angle and attack angle outputs may cause the missile control system become unstable and cannot provide the fast and accurate control. In this paper, the author build up a longitudinal loop model, and design the missile longitudinal loop control system based on the PID controller. Through the simulation test, it draws the conclusions that the PID controller can make the system become stable. At the same time, the determination of the key parameters of aerodynamics variable and velocity can make the foundation and support to the design and development of the missile control system.
\end{abstract}

\section{Introduction}

Nowadays, the modern war puts a higher requirement on missile with high precision, smaller in size, stronger in power and multiple purposes. The missile control and guidance technologies is developing to provide the missile with more stable control performance, better maneuverability and accurate guidance performance, coordinate the conflict between the speed and stability, and increase its anti-interference capacity. The regular controlling system developed in mid 20 century is hardly to satisfy the needs for modern weapons. Researchers are gradually combining the controls of gain scheduling, robust, self-adaptation and variable structure into the missile guidance control. As an important part in missile guidance and control system, the longitudinal loop control system currently available is based on the application of Extended State Observer (ESO) and the theories of $H_{\infty}$ Control and Sliding Mode. However, due to the algorithms for the longitudinal loop controllers are too complex and hard to obtain the necessary state variables in the actual practice, this controlling design has great limitations in its actual application[1-5]. Particularly that this system will become unstable after integrating with the pitching angle feedback. While adding the PID controller into the missile longitudinal loop system can help to set up a deterministic system with the accurate mathematical model, which provides the better robust performance and reliability. On the other hand, the PID controller-based longitudinal loop system can simplified its algorithms and shorten it controlling time. The determined proper PID parameters can make the system stable even with the pitching angle feedback and realize the independence of steady state outputs and missile specifications.

\section{Establishment of Model}

Based on the mathematical model, we select a proper coordinate system to study the longitudinal control of the missile. After that, the relevant transfer function can be deduced from the missile longitudinal loop dynamics model, finally to build up a longitudinal movement control model [6-7]. 


\section{Dynamic Equation}

$$
\begin{aligned}
& \left\{\begin{array}{l}
\frac{d \Delta V}{d t}=\frac{P^{V}-Q^{V}}{m} \Delta V-\frac{P^{\alpha}+Q^{\alpha}}{m} \Delta \alpha-g \cos \gamma \Delta \gamma \\
\frac{d \Delta \gamma}{d t}=\frac{P^{V} \alpha+Y^{V}}{m V_{d}} \Delta V+\frac{P+Y_{\gamma}^{\alpha}}{m V_{d}} \Delta \alpha+\frac{g \sin \gamma}{V_{d}} \Delta \gamma+\frac{Y_{\gamma}^{\delta_{z_{b}}}}{m V_{d}} \Delta \delta_{z}+\frac{Y_{f}}{m V_{d}} \\
\frac{d \Delta r_{b}}{d t}=\frac{M_{z_{b}}^{V}}{I_{z_{b}}} \Delta V+\frac{M_{z_{b}}^{r}}{I_{z_{b}}} \Delta \gamma_{b}+\frac{M_{z_{b}}^{\alpha}}{I_{z_{b}}} \Delta \alpha+\frac{M_{z_{b}}^{\alpha \alpha}}{I_{z_{b}}} \Delta \alpha \alpha_{+} \frac{M_{z_{b}}^{\delta_{z_{b}}}}{I_{z_{b}}} \Delta \delta_{z_{b}}+\frac{M_{z_{f}}}{I_{z_{b}}} \\
\frac{d \theta}{d t}=\Delta r_{b} \\
\Delta \gamma=\Delta \theta-\Delta \alpha
\end{array}\right. \\
& P^{V}: \frac{d P}{d V} \text {, same as other; } \\
& M_{z_{f}} \text { : Disturbance torque; } \\
& Y_{f} \text { : Disturbing force; }
\end{aligned}
$$

$m, \gamma, \alpha, \theta$ : Undisturbed parameter, and served as the function of time. Dots in the variable are the deviation of time. $\mathrm{m}$ stands for the mass of missile; $\mathrm{V}$ for velocity of missile in flight; $\alpha$ for angle of attack; $\gamma$ for inclination angle of ballistic trajectory; $\theta$ for angle of pitch; $\delta_{z_{b}}$ for deflection angle of pitch-control motor; $P$ for engine thrust; $Q$ for head resistance; $Y$ for lift force; $M_{z_{b}}$ for pitching moment; $I_{z_{b}}$ for inertia moment for missile while rotating around Axis $o z_{b}$.

\section{Transfer Function}

Based on Formula (1), the state equation, movement equation of the missile can be obtained with the linear coefficient and solid coefficient under the minimum disturbance.

$$
\left[\begin{array}{l}
\delta \\
\delta \\
\theta
\end{array}\right]=\left[\begin{array}{ccc}
a_{1} & -a_{2} & a_{2} \\
0 & -a_{4} & a_{4} \\
1 & 0 & 0
\end{array}\right]\left[\begin{array}{l}
r \\
\gamma \\
\theta
\end{array}\right]+\left[\begin{array}{ccc}
a_{3} & a_{6} & 0 \\
0 & 0 & a_{8} \\
0 & 0 & 0
\end{array}\right]\left[\begin{array}{c}
\delta_{z} \\
M_{z f} \\
Y_{f}
\end{array}\right]
$$

That is

$$
\begin{aligned}
& \left\{\begin{array}{l}
\hat{\alpha}=A x+B u \\
y=C x
\end{array}\right. \\
& A=\left[\begin{array}{ccc}
a_{1} & -a_{2} & a_{2} \\
0 & -a_{4} & a_{4} \\
1 & 0 & 0
\end{array}\right], \quad B=\left[\begin{array}{ccc}
a_{3} & a_{6} & 0 \\
0 & 0 & a_{8} \\
0 & 0 & 0
\end{array}\right], \quad C=\left[\begin{array}{lll}
1 & 0 & 0 \\
0 & 1 & 0 \\
0 & 0 & 1
\end{array}\right] \\
& u=\left[\begin{array}{lll}
\delta_{z_{b}} & M_{z_{f}} & Y_{f}
\end{array}\right]^{T}, \quad x=\left[\begin{array}{lll}
r_{b} & \gamma & \theta
\end{array}\right]^{T} \\
& a_{1}=M_{z_{b}}^{r_{b}} / I_{z_{b}}\left[1 / \mathrm{s}^{2}\right], \quad a_{2}=M_{z_{b}}^{\alpha \alpha} / I_{z_{b}}\left[1 / \mathrm{s}^{2}\right], \quad a_{3}=M_{z_{b}}^{\delta_{z_{b}} / I_{z_{b}}}\left[1 / \mathrm{s}^{2}\right], \\
& a_{4}=\frac{P+Y^{\alpha}}{m V_{d}}[1 / \mathrm{s}], \quad a_{6}=\frac{1}{I_{z_{b}}}\left[1 / \mathrm{kg} \mathrm{m} \mathrm{s}^{2}\right], a_{8}=\frac{1}{m V_{d}}[1 / \mathrm{kg} \mathrm{s}]
\end{aligned}
$$

Where, the Transfer function is

$$
G(\mathrm{~s})=C(\mathrm{~s} I-\mathrm{A})^{-1} B
$$


In the traditional longitudinal loop system, the pitch rate feedback is considered, so the missile transfer function is described as $G_{\delta_{z_{1}}}^{\delta_{\alpha}}=\frac{K_{c \omega}\left(T_{1 c} s+1\right)}{T_{c}^{2} s^{2}+2 T_{c} \zeta_{c} s+1}$.

While the additional pitch feedback is adopted in the system, according to the Formula (3) above, the following transfer functions can be obtained: Pitch angle transfer function:

$$
G_{\delta_{z_{1}}}^{\Theta}=\frac{K_{c \omega}\left(T_{1 c} s+1\right)}{s\left(T_{c}^{2} s^{2}+2 T_{c} \zeta_{c} s+1\right)}
$$

Trajectory inclination transfer function:

$$
G_{\delta_{z_{1}}}^{\gamma}=\frac{K_{c \omega}}{s\left(T_{c}^{2} s^{2}+2 T_{c} \zeta_{c} s+1\right)}
$$

Attach angle transfer function:

$$
G_{\delta_{z_{1}}}^{\theta}=\frac{K_{c \alpha}}{T_{c}^{2} s^{2}+2 T_{c} \zeta_{c} s+1}
$$

where $K_{c \omega}$ served as transfer coefficient; $T_{1 c}$ as aerodynamic force constant; $T_{c}$ as time constant; $\zeta_{c}$ as relative damping factor.

\section{Longitudinal Control Loop System}

The missile longitudinal control loop system is mainly composed of gyroscope, steering control system and controlling algorithms[8-10]. Its simplified model is shown in Figure 1 below.

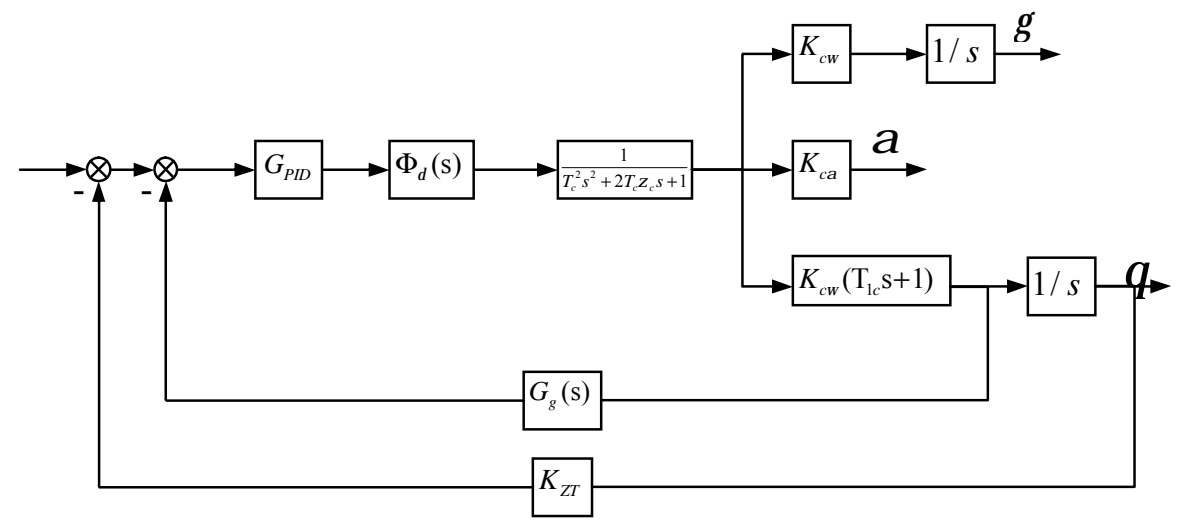

Figure 1:Structure of Longitudinal Closed-loop Control System

In Figure 1, $G_{P I D}(s)$ is regarded as the transfer function of PID controller, $\Phi_{\delta}(s)$ as steering control system transfer function, and $K_{Z T}$ as Angular Speed Gyroscope transfer function. Due to the time constant for missile body will normally much larger than the time constants of steering system and gyroscope, $\Phi_{\delta}(s)$ and $G_{g}(s)$ can be approximated to the corresponding part. That is: $\Phi_{\delta}(s) \approx K_{\delta}=3.6, G_{g}(s) \approx K_{g}=0.056$.

\section{PID Controller Design and Stimulation Results}

\section{Determination of PID Parameters}

The projectile transfer function occurred in the slight damped oscillation process, so its amplitude frequency is featuring with resonance peak and phase frequency drops rapidly. In order to make the system stable and isolate the steady state outputs and missile parameters, the PID controller needs to provide the positive phase angle to compensate the negative phase angle of 
missile. In this way, the phase angle can be widen to meet the requirements[11-13].

To simplify the parameter design, $G_{P I D}=K_{P}\left(\frac{T_{I} T_{D} s^{2}+T_{I} s+1}{T_{I} s}\right)$,

and $T_{1}, T_{D}$ should satisfy $\left\{\begin{array}{l}T_{I} T_{D}>T_{c}^{2} \\ T_{I}>2 \zeta_{c} T_{c}\end{array}\right.$.

Where $K_{P}$ is the proportional gain, a turning parameter. A high proportional gain can enlarge the open-loop gain and reduce the steady-state error to make the system more sensitive. However, the system will become unstable. $T_{I}$ is the integral time, which can upgrade the system type and increase the system stability. However, it will delay the signal response. $T_{D}$ is the derivative time taking its effect during the dynamic process. It can reflect the variation trend of the signal and produce the correction signal in advance. However, if this derivative time is determined improperly, the regulating time will become longer.

We design the PID parameters as the way below:

To make $\left(\mathrm{T}_{c}^{2} \mathrm{~s}^{2}+2 \mathrm{~T}_{c} \zeta_{c} \mathrm{~s}+1\right)\left(\mathrm{T}_{\delta}^{2} \mathrm{~s}^{2}+2 \mathrm{~T}_{\delta} \zeta_{\delta} \mathrm{s}+1\right)$,

the denominator of product of the pitch rate transfer function times steering control system transfer function can be compensated with $T_{I} T_{D} s^{2}+T_{I} s+1$.

That is to neglect the higher order term of $\left(T_{\delta}^{2} s^{2}+2 T_{\delta} \zeta_{\delta} s+1\right)\left(T_{c}^{2} s^{2}+2 T_{c} \zeta_{c} s+1\right)$,

Then $\left(T_{\delta}^{2}+T_{c}^{2}+4 \zeta_{\delta} T_{\delta} \zeta_{c} T_{c}\right) s^{2}+\left(2 \zeta_{\delta} T_{\delta}+2 \zeta_{c} T_{c}\right) s+1$,

Then $T_{I} T_{D} s^{2}+T_{I} s+1=\left(T_{\delta}^{2}+T_{c}^{2}+4 \zeta_{\delta} T_{\delta} \zeta_{c} T_{c}\right) s^{2}+\left(2 \zeta_{\delta} T_{\delta}+2 \zeta_{c} T_{c}\right) s+1$,

Therefore $\left\{\begin{array}{l}T_{I} T_{D}=T_{\delta}^{2}+T_{c}^{2}+4 \zeta_{\delta} T_{\delta} \zeta_{c} T_{c} \\ T_{I}=2 \zeta_{\delta} T_{\delta}+2 \zeta_{c} T_{c}\end{array}\right.$

For the parameters of a type a missile at a certain time in flight, according to the amplitude-frequency characteristic and phase-frequency characteristic, $K_{P}=90, T_{I}=8.5, T_{D}=0.34$.

\section{Expected Results of PID Controller-based System}

First of all, it should be a stable system. Secondly, if the given attitude angle is $\theta_{\mathrm{k}}$ which be input with unit step, the control surface will deflect upward $\delta_{z_{b}}$ degree. Therefore the missile nose can pitch up and become an angle of $\theta$, which produces a positive attack angle gain $\alpha$. At the meantime, since the angle of attack become larger, the life force is increased accordingly, and the inclination angle $\gamma$ is enlarged gradually till achieving $\theta=\gamma=\theta_{k}, \alpha=0$. Finally the missile will rise up in a new movement.

\section{Simulation Results}

We carry out the stimulation via Matlab. The stimulation figures of pitch angle step function response curves in the longitudinal loop before and after integrating the PID controller are shown as Figure 2 and Figure 3.

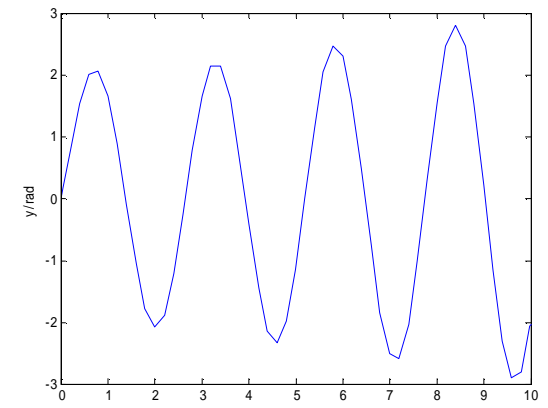

Figure 2:Pitch angle step function response curve WITHOUT PID Controller

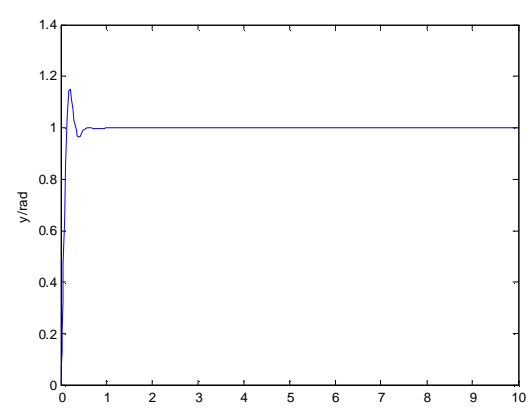

Figure 3DZ:Pitch angle step function response curve WITH PID Controller 
Comparing the two curves above, we find that the PID controller-based missile longitudinal loop control system is stable.

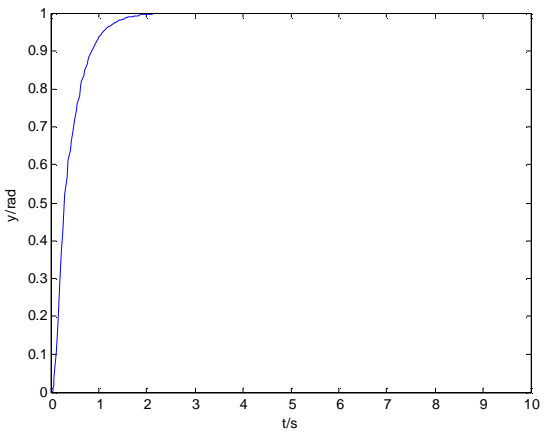

Figure 4:Trajectory inclination step function response curve

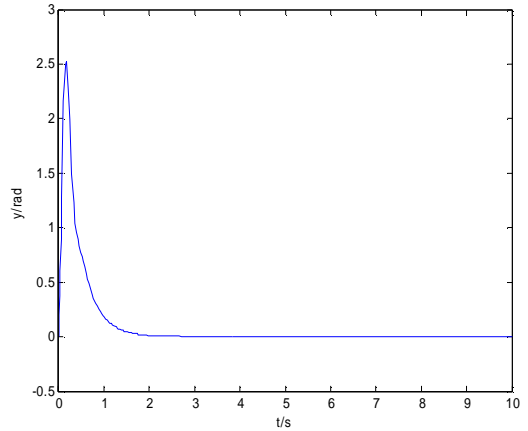

Figure 5:Attack angle step function response curve

Compared to the traditional missile longitudinal loop system, this PID controller-based system further integrates the outputs of trajectory inclination angle and attack angle. A series of key parameters such as the determination of velocity by trajectory inclination angle and the determination of aerodynamics variable by inclination angle, provide the necessary and solid support for the operation of the whole system control.

\section{Summary}

We combine the PID controller into the traditional longitudinal loop system of the missile design. Through the stimulation we done, the PID controller-based longitudinal loop control system can improve the stability of the system after having the pitch angle feedback. This stimulation verifies the effectiveness and feasibility of the PID controller-based system. On the other hand, the design of PID controller is simpler and easy to take effect, which further demonstrates the high effectiveness of the new system. At this stage, there are no proposed improvements to the PID controller yet, which will be further studied in the later stage.

\section{Acknowledgement}

The paper is supported by Project of Science and Technology of Liaoning . (No. 2013405003)

\section{References}

[1] Dong Fei-yao,Lei Hu-min,Shao Lei,Zhang Jing-peng.Design of control system for missile longitudinal plane based on extended state observer[J].Systems Engineering and Electronices.2012,Vol.34,NO.1,pp:125-128.

[2]Tan F, Duan G, Zhao L. Robust controller design for autopilot of a BTT missile[C]//Intelligent Control and Automation, 2006. WCICA 2006. The Sixth World Congress on. IEEE, 2006, 2: 6358-6362.

[3]Hall C E,Shtessel Y B.Sliding mode disturbance observer-based control for a reusable launch vehicle[C]//Proc. Of the AIAA Guidance Navigation and Control Conference and Exhibit,2005:1-26.

[4] Ma Sa-sa,Zhang Ji-wei,Cai Shen-chun,Feng Qian.Application of QFT for missile flight control[J].Transactions of Beijing Institute of Technology.2013,Vol. 33, NO.2, pp:155-159.

[5] Chen Wei-dong,Chen Yang-yang,Xue Zhi-xia,Xu Jiang-tao.Deisign of Control System for Cruise Missile Based on Double Sliding Mode Variable Stracture[J].Aeronautical computing Technique.2013,Vol.43,NO.1,pp:4-8. 
[6]Shi Zhen, Yao Xu-liang,Yu Xiu-fang.Vehicle Control Systems[M]. Tsinghua University Press.2008.

[7] Hu shou-song.Automatic Control Theory[M].Beijing Science Press.2005

[8] Bo Zhang, Shushan Wang, Mengyu Cao, et al. Impacts of Deflection Nose on Ballistic Trajectory Control Law, Mathematical Problems in Engineering, Volume 2014,Article ID 984840,6 pages.(SCI, Accession Number: WOS: 000332969500001)

[9] Qian Xing-fang,Lin Rui-xiong,Zhao Ya-nan.Flight mechanics of missle[M].Press of Being Institute of Technologu.2008

[10] Zhang Bo, Xu Yuxin, Cao Mengyu, etal. Design and Research of Wind Tunnel Test for Deflectable Nose[J]. Laboratory Research and Exploration, 2014, 33(4):18-21.

[11]JIANG Xiu-qiang,TAN Fei , Missile longitudinal Loop Control and Simulation Based on PID with Correction, Electronic Design Engineering,2010,Vol.18, No. 11, pp:124-127

[12] Woo Zhi-Wei, Chung Hung-Yuan, Lin Jin-Jye. A PID type fuzzy controller with self-tuning scaling factors[J]. Fuzzy Sets and Systems, 2010, 115(3): 321-326.

[13] Bo Zhang, Shushan Wang, Mengyu Cao, et al. Design and Research of Wind Tunnel Test for Deflectable Nose, Applied Mechanics and Materials [J]. Vols. 423-426 (2013): pp 2063-2067. (EI, Accession Number: 20134416915412) 